\title{
Novel MSH2 splice-site mutation in a young patient with Lynch syndrome
}

\author{
RAFFAELLA LICCARDO $^{1}$, MARINA DE ROSA ${ }^{1}$, PAOLA IZZO $^{1,2}$ and FRANCESCA DURATURO ${ }^{1}$ \\ ${ }^{1}$ Department of Molecular Medicine and Medical Biotechnology, \\ University of Naples 'Federico II'; ${ }^{2}$ CEINGE, Biotecnologie Avanzate, I-80131 Naples, Italy
}

Received November 6, 2017; Accepted February 27, 2018

DOI: $10.3892 / \mathrm{mmr} .2018 .8752$

\begin{abstract}
Lynch Syndrome (LS) is associated with germline mutations in one of the mismatch repair $(M M R)$ genes, including MutL homolog 1 (MLH1), MutS homolog 2 (MSH2), MSH6, PMS1 homolog 2, mismatch repair system component (PMS2), MLH3 and MSH3. The mutations identified in $M M R$ genes are point mutations or large rearrangements. The point mutations are certainly pathogenetic whether they determine formation of truncated protein. The mutations that arise in splice sites are classified as 'likely pathogenic' variants. In the present study, a novel splicing mutation was identified, (named c.212-1g>a), in the $M S H 2$ gene. This novel mutation in the consensus splice site of MSH2 exon 2 leads to the loss of the canonical splice site, without skipping in-frame of exon 2; also with the formation of 2 aberrant transcripts, due to the activation of novel splice sites in exon 2. This mutation was identified in a young patient who developed colon cancer at the age of 26 years and their belongs to family that met the 'Revised Amsterdam Criteria'. The present study provided insight into the molecular mechanism determining the pathogenicity of this novel $M S H 2$ mutation and it reaffirms the importance of genetic testing in LS.
\end{abstract}

\section{Introduction}

The identification of mutations in genes that determine a high risk of developing hereditary colorectal cancer (CRC) has allow to performed genetic testing to identify subjects with strong predisposition to cancer (1). The hereditary CRC syndromes are divided in polyposis, as FAP, MAP, PHTS (2-4), and in non-polyposis, as Lynch syndrome (LS) (5). The LS is an autosomal dominant syndrome; besides CRC, the phenotypic

Correspondence to: Dr Francesca Duraturo, Department of Molecular Medicine and Medical Biotechnology, University of Naples 'Federico II', Via Pansini, 5, I-80131 Naples, Italy E-mail: duraturo@dbbm.unina.it; francesca.duraturo@unina.it

Key words: Lynch syndrome, hereditary non-polyposis colorectal cancer, MutS homolog 2 gene, splice-site mutation, aberrant transcripts, novel variant MutS homolog 2 gene spectrum of LS includes other primary tumors, as cancer of the stomach, endometrium, biliary and pancreatic system, and urinary tract. The 'Revised Amsterdam Criteria' represent the diagnostic guidelines designed to identify LS families. Subsequently, Bethesda guidelines were developed to improve identification of LS patients $(5,6)$.

The LS is associated with germline mutations in one of the mismatch repair $(M M R)$ genes, including MutL homolog 1 (MLH1), MutS homolog 2 (MSH2), MSH6, PMS1 homolog 2, mismatch repair system component (PMS2), MLH3 and MSH3 (7-10). The loss of function of one MMR protein prevents to repair's complex to work properly and this determines a genetic instability known as microsatellite instability (MSI) at somatic level (11). The mutations in $M M R$ genes are point mutations or large rearrangements $(7,12,13)$. Most of point mutations determine formation of truncated protein through nonsense mutations, insertions or deletions; many often, substitutions of a single nucleotide (as in missense and silent mutations) may also create a truncated protein (14). Indeed, some exonic and intronic variants create or disrupt splice sites and consequently, this leads the creation of aberrant splicing mRNA transcripts (7). All these genetic variants in $M M R$ genes altering the protein function are considered to be 'pathogenic'.

In this study, we have identified and characterized a novel MSH2 gene splicing mutation in a LS young patient.

\section{Materials and methods}

Patient history. Our proband is a 28-year-old male. He underwent partial colon resection (proximal descending and transverse colon) for a poorly differentiated adenocarcinoma in transverse colon diagnosed at the age of 26 years. Subsequenty, at 27 years of age, he developed sigmoid tubular adenomatous polyp, which was resected. The proband's family history was also positive for colorectal and extra-colonic cancer. A detailed pedigree is shown in Fig. 1.

Sample from our patient was collected after being granted authorisation from the Ethics Committee 'Comitato etico per le attività Biomediche-Carlo Romano' of the University of Naples Federico II (Naples, Italy; protocol no. 120/10). Once the authorisation has been obtained the study has received ethical approval, and the participant informed and written consent has been obtained. 
Furthermore, as negative controls we collected 100 healthy samples from Clinical Department of Laboratory Medicine of our Hospital (Federico II of Naples).

Isolation of genomic DNA. The genomic DNA was extracted from peripheral blood lymphocytes and from paraffin-embedded tumor tissue of our patient. Total genomic DNA was extracted from $4 \mathrm{ml}$ peripheral blood lymphocytes using a BACC2 Nucleon kit (Amersham; GE Healthcare, Chicago, IL, USA). For each paraffin block, five $20-\mu$ m-thick sections were cut and collected in a $1.5-\mathrm{ml}$ micro tube and $1 \mathrm{ml}$ xylene was added to each tube and kept at room temperature for $20 \mathrm{~min}$ to remove the paraffin completely, according to the protocol described by Duraturo et al (7). Subsequently, the somatic DNA was extracted using a BACC2 Nucleon kit.

Microsatellite analysis and V600E BRAF mutation analysis. Microsatellite instability was tested on paired samples from lymphocyte DNA and from paraffin-embedded tumor colon tissues. The MSI status was evaluated using the CC-MSI kit (AbAnalitica, Padova, Italy) and subsequent capillary electrophoresis analysis using an ABI 3130 Prism (Applied Biosystems; Thermo Fisher Scientific, Inc., Waltham, MA, USA), as previously described (7). For V600E genotyping, genomic DNA extracted from paraffin-embedded tumor tissue and blood lymphocytes were amplified using customized primer pair, (15F-5'-TGCTTGCTCTGATAGGAAAAT GAGA-3'- and 15R-5'-GGCCCTGAGATGCTGCTGAG-3'-), and sequenced in both the forward and reverse directions using an ABI 3100 Genetic Analyser (Applied Biosystems; Thermo Fisher Scientific, Inc.).

Mutation analysis: Amplification, dHPLC and sequencing. All MLH1 (NM_000249) and MSH2 (NM_000251.2) exons were amplified, including intron-exon boundaries, on DNA extracted from blood lymphocytes of our patient, using customized primer sets. Prior to dHPLC analysis, the polymerase chain reaction (PCR) products were run on an 1-2\% agarose gel to check for unspecific amplicons. A Transgenomic Wave DNA Fragment Analysis System (3500 HT) was used to perform dHPLC analysis (Transgenomic Inc., Omaha, NE, USA) using personal methods, available on request; subsequently, genomic DNA was re-amplified and sequenced in both the forward and reverse directions using an ABI 3100 Genetic Analyser (Applied Biosystems; Thermo Fisher Scientific, Inc.).

In silico analysis. We analyzed the novel variant detected in this study by the Human Splicing Finder (HSF) software (http://www.umd.be/HSF/) (15), a tool designed to predict the effects of mutations on splicing signals or to identify splicing motifs in human sequences, as previously described.

Reverse transcription (RT)-PCR MSH2 cDNA fragment. Total RNA was extracted from lymphocytes of our patient and three normal controls using TRIzol reagent (Invitrogen; Thermo Fisher Scientific, Inc.) by standard procedure. cDNA was synthesised using SuperScript II RT (Invitrogen; Thermo Fisher Scientific, Inc.) according to the manufacturer's instructions. PCR amplification reactions of the cDNA fragment of $563 \mathrm{bp}$, including the exons 1-2-3 of $\mathrm{MSH} 2$ gene was performed using customized primer pair, (1F-5'-CTTCGTGCGCTTCTT TCAG-3' and 1R-5'-TGTTTTACCCGGAGGAGAGA-3'). Amplified fragments were visualized on $1 \%$ agarose gel and a $8 \%$ polyacrilamide gel. Each band was excised from polyacrilamide gel and re-suspended only in $30 \mu \mathrm{l}$ water, over-night. Then, $1 \mu \mathrm{l}$ was re-amplified and subsequently sequenced using the same primer pair.

\section{Results}

First, we performed MSI analysis on our proband and a MSI-H status was detected in DNA extracted from tumour tissues, with instability of all nucleotide markers analyzed and complete absence of BAT26 mononucleotide. No V600E mutation in $B R A F$ gene was identified. Subsequently, all $M L H 1$ and $M S H 2$ exons were analysed by DHPLC and the altered exonic fragments were only sequenced. In this manner, we identified a novel mutation in the $\mathrm{MSH} 2$ gene that determined a nucleotide substitution $(\mathrm{g}>\mathrm{a})$ in the acceptor site, upstream at exon 2. The mutation that is named c. $212-1 \mathrm{~g}>\mathrm{a}$, has not been reported before in the international database of InSiGHT-Group (http://www.insight-group.org/) and it was not detected in 100 healthy controls analyzed, Fig. 2A. In silico analysis carried out using the HSF software showed that this mutation altered the wild type acceptor site and most probably it created a new acceptor site, Fig. 2B. The analysis by PCR of the $M S H 2$ cDNA fragment including the exons 1-2-3 of this gene confirmed this computational data. In particular, abnormal band was showed by this analysis, Fig. 3A. Each amplification product visualized on the polyacrilamide $8 \%$ gel has been extracted and sequenced, as described in Materials and Methods. Two bands revealed two splicing isoforms of $M S H 2$ mRNA determining a skipping of 80 and 85 bp, respectively [(c.212_c.292) and (c.212_c.297)], Fig. 3B and C. Both two transcripts determined a frameshift with premature stop codon and consequently, the formation of a truncated protein (p.Gly71Valfs*2 and p.Gly71Glufs ${ }^{*} 75$, respectively). Therefore, this mutation is considered as pathogenetic. No large rearrangements in $M L H 1$ and $M S H 2$ genes and no other point mutations in $M L H 1$ and MSH6 genes were identified in our patient.

\section{Discussion}

Genetic testing of $M M R$ genes has been widely applied to aid the diagnosis of LS. So far, many pathogenetic mutations were identified in these genes, in particular in $\mathrm{MLH1}$ and $\mathrm{MSH} 2$.

In this study, we identified a novel germline variant, $\mathrm{MSH} 2$ c.212-1g>a, which is a variant that affects the correct mechanism of splicing. This variant is located in acceptor splice site (-1) upstream of exon 2 in $M S H 2$ and it created two new splice acceptor site to 80 and 85 bases respectively, downstream of the primary splice acceptor site. Thus, this mutation didn't determine the skipping in-frame of exon 2 but it promoted the formation of two aberrant splicing transcripts with the loss of 80 and $85 \mathrm{bp}$, respectively. Both this splicing isoforms determined a frameshift of $M S H 2$ mRNA with consequent premature stop codon, 2 and 75 codons after, respectively. The formation of a premature stop codon located within and not at the end of the transcript increases the probability that non-sense mediated decay (NMD) might be involved in 


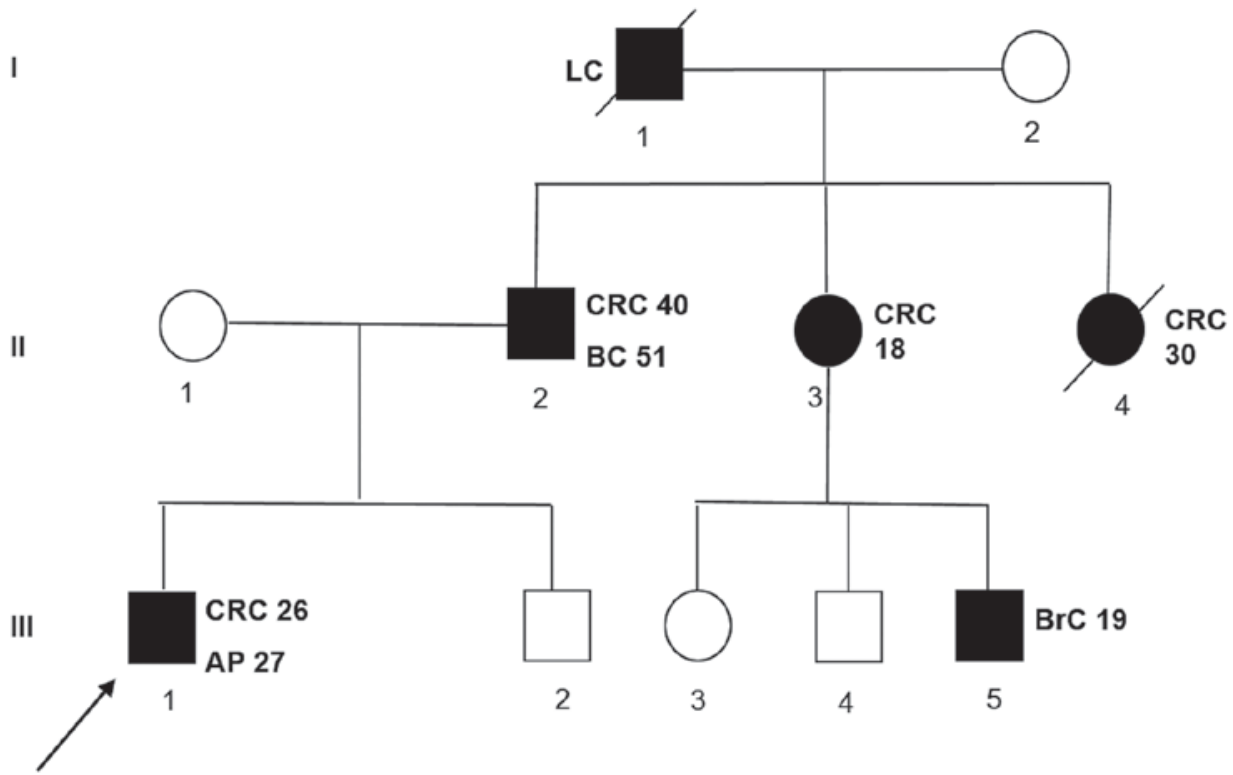

Figure 1. Family pedigree of the patient with a novel MutS homolog 2 gene mutation. The numbers next to each diagnosis denote the age at onset. Symbols and abbreviations used are denoted as follows: Arrow, index case (proband); symbols with diagonal lines, succumbed; black symbol, CRC or tumors associated with Lynch syndrome; CRC, colorectal cancer; LC, lung cancer; BC, bladder cancer; BrC, brain cancer; AP, adenomatous polyp; squares, males; circles, females.

A

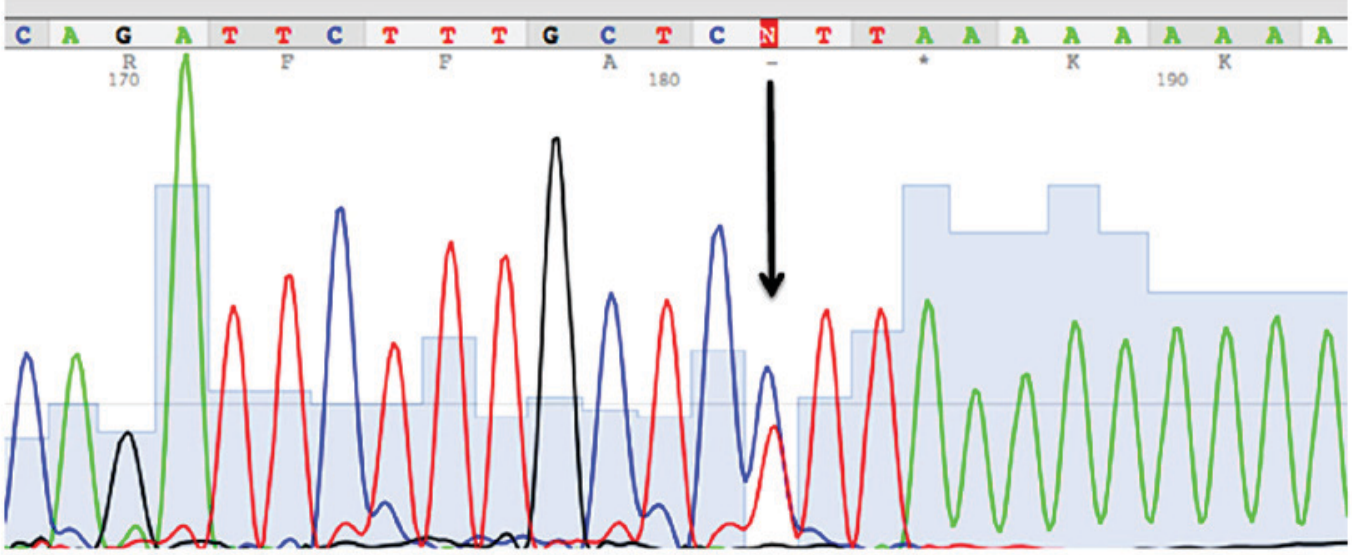

B

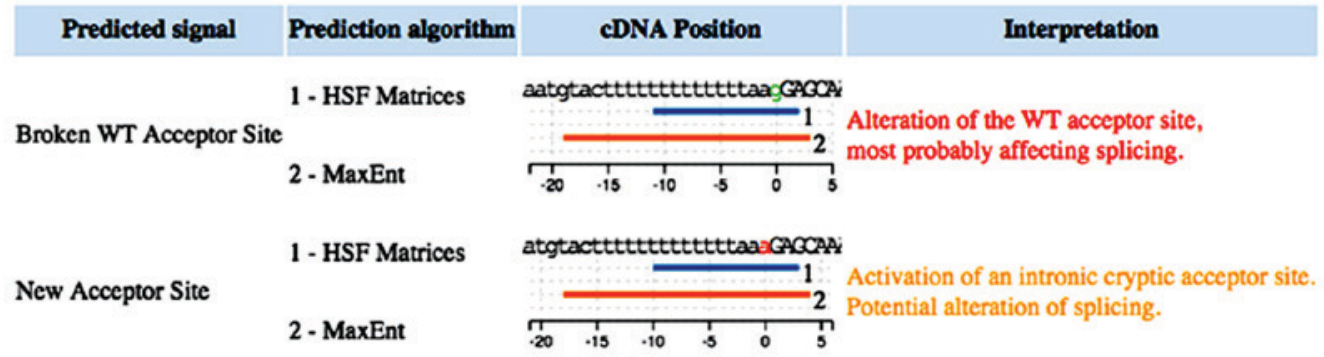

Figure 2. (A) Electropherogram showing the novel mutation identified in the acceptor splice site of exon 2 in MutS homolog 2 gene, c. 212-1g>a. (B) In silico analysis of this novel variant using HSF software. HSF, Human Splicing Finder; WT, wild type.

degrading aberrant transcripts (16). Anyhow, two aberrant transcripts likely formed a truncated protein thus not functional. This result was concordant with the MSI-high status (it showed instability of all repetitive sequences analyzed) and with complete absence of BAT26 markers on DNA extracted from colon cancer specimens of our patient. It is known that the variants around the splicing donor and acceptor sites alter the correct splicing mechanism (17). According to the InSiGHT databases (http://www.insight-group.org/), the variants that fall into the splice site, donor or acceptor $( \pm 1$ or \pm 2$)$ are classified 


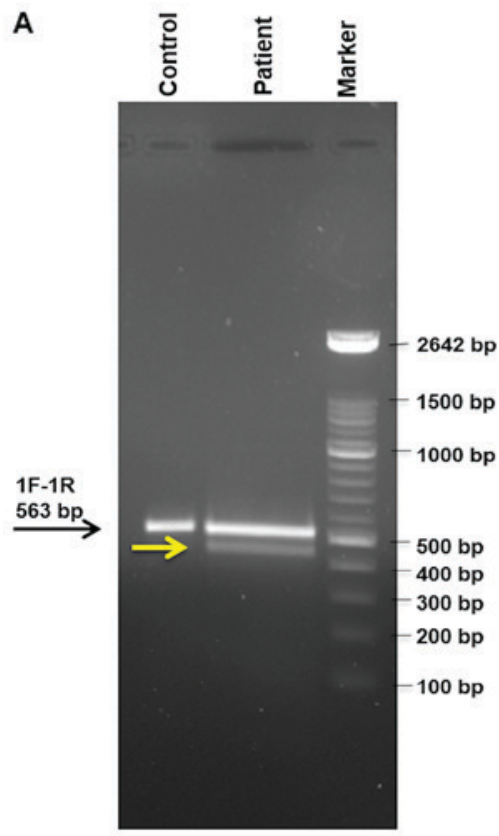

B

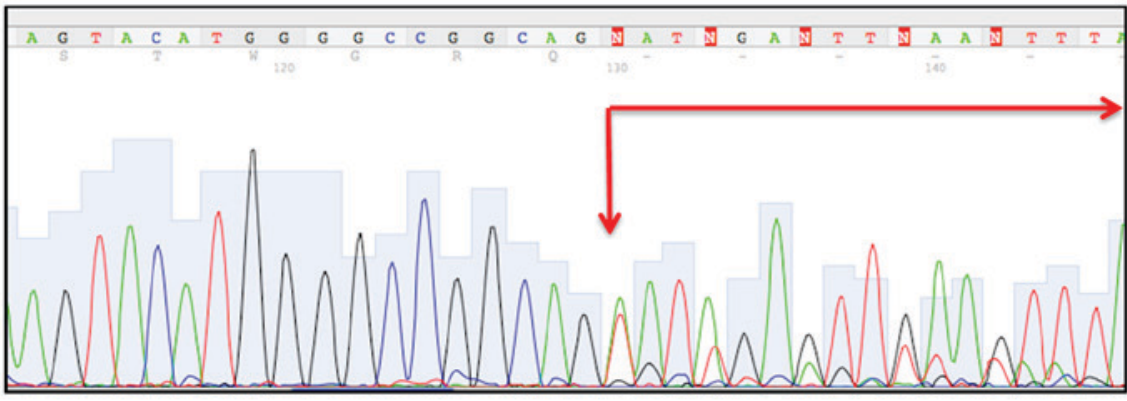

C

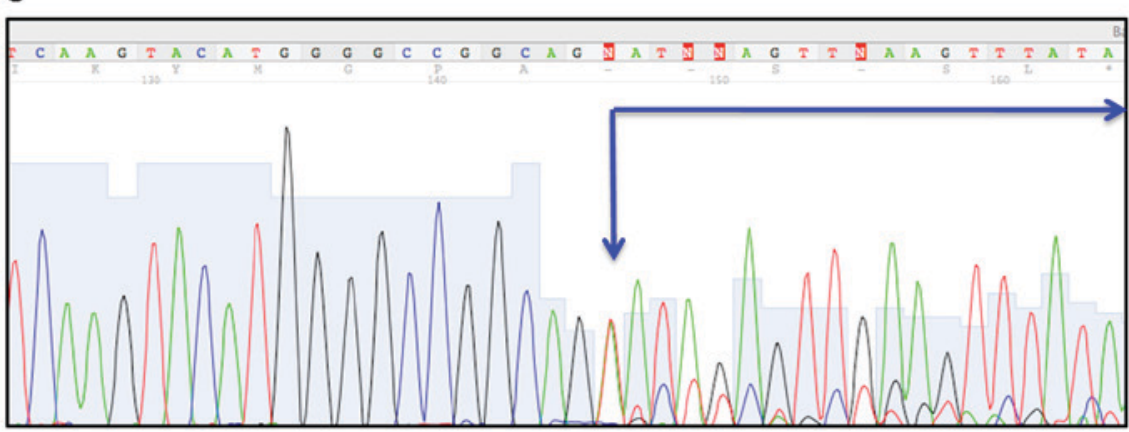

Figure 3. Analysis of $M S H 2$ cDNA in the proband carrying the novel variant, c.212-1 g>a. (A) $1 \%$ agarose gel of polymerase chain reaction fragment with a size of $563 \mathrm{bp}$, including the exons 1-2-3 of $\mathrm{MSH} 2$ gene, on cDNA of the patient and one of the normal controls; abnormal aberrant splicing was observed in the proband. The black arrow indicates the amplified fragment; the yellow arrow shows the band that corresponded to aberrant splicing isoforms. (B) Electropherogram of aberrant transcript of $M S H 2$ cDNA showing the loss of $80 \mathrm{bp}$, named c.212_c.292. (C) Electropherogram of aberrant transcript of MSH2 cDNA showing the loss of 85 bp, named c.212_c.297. MSH2, MutS homolog 2; bp, base pairs.

as 'likely pathogenic' variants. The most of these variants in canonical splice site determines the skipping in-frame of relative exon. Interestingly, the molecular characterization of our novel variant, the c212-1g>a in $M S H 2$ gene showed that it created two aberrant splicing transcripts [(c.212_c.292) and (c.212_c.297)] and that both determined the formation of protein truncated (p.Gly71Valfs*2 and p.Gly71Glufs*75).

This novel MSH2 mutation was identified in a 28 -year-old patient who developed an adenocarcinoma in transverse colon diagnosed at the age of 26 years and, subsequently he developed sigmoid tubular adenomatous polyp at 27 years of age. Our patient's family history was also positive for colorectal and extra-colonic cancer and met the 'Revised Amsterdam Criteria', Fig. 1. In this family, there were two cases of colon cancers early onset (II-3 and II-4 subjects) and several cases of extracolic cancers. Indeed, the subject III-5 developed a brain cancer at 18 years of age, the proband's father (II-1) has developed a bladder cancer beyond colon cancer and the grandfather (I-1) a lung cancer. Unfortunately, we were not able to performed genetic testing for other affected subjects of this family due to their limited availability. However, this variant, that is considered to be 'pathogenic' is likely responsible of LS phenotype in this family.

It remains to be clarified whether $M S H 2$ mutation alone induces the occurrence of LS-related cancers and very early onset of these cancers, in this family. Literature review indicated that genetic disorder and dietary and/or environment factors had synergistic effect in promoting cancer initiation in $M S H 2$-defective individuals (17). For example, it was reported that germline ablation of SMUG1 DNA glycosylase causes loss of 5-hydroxymethyluracil- and UNG-backup uracil-excision activities and increases cancer predisposition of Ung-/-Msh2-/- mice (18); as it has also been shown that interaction between microbiota and dietary factors tends to reduce the occurrence of CRC and other cancers in APC (Min/+)MSH2(-/-) mice (19). Therefore, this suggested that other genetic instabilities could increase the risk to develop LS-releted cancers and to anticipate age onset MSH2-defective resultant cancers.

In conclusion, in this study we have identified and characterized a novel splicing mutation in $\mathrm{MSH} 2$ gene. In particular, we investigated how one novel mutation into the consensus splice site of $\mathrm{MSH} 2$ exon 2 leads the formation of two aberrant transcripts, due to activation of new splice sites inside of exon 2. Moreover, in the light of recent literature data according to which molecular characterization of cancer-associated mutations can provide valuable information on disease prognosis and patient response to therapy (20) this study reaffirms the importance to identify pathogenic mutations in LS families to facilitate pre-symptomatic diagnosis and to improve therapeutic pathway in order to promote a personalized medicine.

\section{Acknowledgements}

Not applicable. 


\section{Funding}

The present study was supported by the Campania Regional Authority [granted to CEINGE, Biotecnologie Avanzate (Naples, Italy; 2010-2012 POR Campania, grant no. fSe2007-2013)].

\section{Availability of data and materials}

The datasets used and analyzed during the current study are available from the corresponding author on reasonable request.

\section{Authors' contributions}

FD and PI conceived and designed the experiments. RL performed the experiments. FD and MDR analyzed the data. FD and MDR interpreted the data, and FD wrote the first draft of the manuscript. FD and RL agreed with the results and conclusions of the manuscript. FD developed the structure and arguments for the paper. RL, MDR and PI made critical revisions. All authors reviewed and approved the final manuscript.

\section{Ethics approval and consent to participate}

The present study was approved by the Ethics Committee of 'Comitato etico per le attività Biomediche-Carlo Romano' of the University of Naples Federico II (Naples, Italy; protocol no. 120/10), and written informed consent was obtained from all participants.

\section{Consent for publication}

Written informed consent was obtained from all participants.

\section{Competing interests}

The authors declare that they have no competing interests.

\section{References}

1. De Rosa M, Pace U, Rega D, Costabile V, Duraturo F, Izzo P and Delrio P: Genetics, diagnosis and management of colorectal cancer (Review). Oncol Rep 34: 1087-1096, 2015.

2. Dodaro C, Grifasi C, Florio J, Santangelo ML, Duraturo F, De Rosa M, Izzo P and Renda A: The role of mutation analysis of the APC gene in the management of FAP patients. A controversial issue. Ann Ital Chir 87: 321-325, 2016.

3. De Rosa M, Galatola M, Borriello S, Duraturo F, Masone S and Izzo P: Implication of adenomatous polyposis coli and MUTYH mutations in familial colorectal polyposis. Dis Colon Rectum 52: 268-274, 2009

4. Paparo L, Rossi GB, Delrio P, Rega D, Duraturo F, Liccardo R, Debellis M, Izzo P and De Rosa M: Differential expression of PTEN gene correlates with phenotypic heterogeneity in three cases of patients showing clinical manifestations of PTEN hamartoma tumour syndrome. Hered Cancer Clin Pract 11: 8, 2013.

5. Liccardo R, De Rosa M, Izzo P and Duraturo F: Novel implications in molecular diagnosis of lynch syndrome. Gastroenterol Res Pract 2017: 2595098, 2017.
6. Giardiello FM, Allen JI, Axilbund JE, Boland CR, Burke CA, Burt RW, Church JM, Dominitz JA, Johnson DA, Kaltenbach T, et al: Guidelines on genetic evaluation and management of Lynch syndrome: A consensus statement by the US multi-society task force on colorectal cancer. Gastroenterology 147: 502-526, 2014.

7. Duraturo F, Liccardo R, Cavallo A, De Rosa M, Rossi GB and Izzo P: Multivariate analysis as a method for evaluating the pathogenicity of novel genetic MLH1 variants in patients with colorectal cancer and microsatellite instability. Int J Mol Med 36: 511-517, 2015.

8. Duraturo F, Liccardo R, Cavallo A, De Rosa M, Grosso M and Izzo P: Association of low-risk MSH3 and MSH2 variant alleles with Lynch syndrome: Probability of synergistic effects. Int J Cancer 129: 1643-1650, 2011.

9. Liccardo R, De Rosa M, Rossi GB, Carlomagno N, Izzo P and Duraturo F: Incomplete segregation of MSH6 frameshift variants with phenotype of lynch syndrome. Int J Mol Sci 18: pii: E999, 2017

10. Duraturo F, Liccardo R and Izzo P: Coexistence of MLH3 germline variants in colon cancer patients belonging to families with Lynch syndrome-associated brain tumors. J Neurooncol 129: 577-578, 2016.

11. Gelsomino F, Barbolini M, Spallanzani A, Pugliese G and Cascinu S: The evolving role of microsatellite instability in colorectal cancer: A review. Cancer Treat Rev 51: 19-26, 2016.

12. Duraturo F, Cavallo A, Liccardo R, Cudia B, De Rosa M, Diana $G$ and Izzo P: Contribution of large genomic rearrangements in Italian Lynch syndrome patients: Characterization of a novel alu-mediated deletion. Biomed Res Int 2013: 219897, 2013.

13. Cudia B, Liccardo R, Di Carlo G, Damiano G, Ignazio A, Monte L, Izzo P and Duraturo F: Clinical and anamnestic evaluation rôle for the diagnosis and treatment of families affected by lynch syndrome. Case report and review of the literature. Eur J Oncol 19: 265-271, 2014.

14. Van der Klift HM, Jansen AM, van der Steenstraten N, Bik EC, Tops CM, Devilee P and Wijnen JT: Splicing analysis for exonic and intronic mismatch repair gene variants associated with Lynch syndrome confirms high concordance between minigene assays and patient RNA analyses. Mol Genet Genomic Med 3: 327-345, 2015.

15. Desmet FO, Hamroun D, Lalande M, Collod-Béroud G, Claustres $\mathrm{M}$ and Béroud $\mathrm{C}$ : Human splicing finder: An online bioinformatics tool to predict splicing signals. Nucleic Acids Res 37: e67, 2009.

16. De Rosa M, Morelli G, Cesaro E, Duraturo F, Turano M, Rossi GB, Delrio P and Izzo P: Alternative splicing and nonsense-mediated mRNA decay in the regulation of a new adenomatous polyposis coli transcript. Gene 395: 8-14, 2007.

17. Hu H, Li H, Jiao F, Han T, Zhuo M, Cui J, Li Y and Wang L: Association of a novel point mutation in MSH2 gene with familial multiple primary cancers. J Hematol Oncol 10: 158, 2017.

18. Kemmerich K, Dingler FA, Rada C and Neuberger MS: Germline ablation of SMUG1 DNA glycosylase causes loss of 5-hydroxymethyluracil- and UNG-backup uracil-excision activities and increases cancer predisposition of Ung-/-Msh2-/- mice. Nucleic Acids Res 40: 6016-6025, 2012.

19. Belcheva A, Irrazabal T, Robertson SJ, Streutker C, Maughan H, Rubino S, Moriyama EH, Copeland JK, Surendra A, Kumar S, et al: Gut microbial metabolism drives transformation of MSH2-deficient colon epithelial cells. Cell 158: 288-299, 2014.

20. De Rosa M, Rega D, Costabile V, Duraturo F, Niglio A, Izzo P, Pace U and Delrio P: The biological complexity of colorectal cancer: Insights into biomarkers for early detection and personalized care. Therap Adv Gastroenterol 9: 861-886, 2016.

This work is licensed under a Creative Commons Attribution-NonCommercial-NoDerivatives 4.0 International (CC BY-NC-ND 4.0) License. 